\section{oluo mental}

Berenzon Gorn, Shoshana; Galván Reyes, Jorge; Saavedra Solano, Nayelhi; Bernal Pérez, Pilar; Mellor-Crummey, Lauren; Tiburcio Saínz, Marcela

Exploración del malestar emocional expresado por mujeres que acuden a centros de atención primaria de la Ciudad de México. Un estudio cualitativo

Salud Mental, vol. 37, núm. 4, julio-agosto, 2014, pp. 313-319

Instituto Nacional de Psiquiatría Ramón de la Fuente Muñiz

Distrito Federal, México

\section{Salud Mental}

perezrh@imp.edu.mx

Instituto Nacional de Psiquiatría Ramón de la

Fuente Muñiz

México

Disponible en: http://www.redalyc.org/articulo.oa?id=58231853005

Cómo citar el artículo

- Número completo

- Más información del artículo

- Página de la revista en redalyc.org 


\title{
Exploración del malestar emocional expresado por mujeres que acuden a centros de atención primaria de la Ciudad de México. Un estudio cualitativo
}

\author{
Shoshana Berenzon Gorn, ${ }^{1}$ Jorge Galván Reyes, ${ }^{1}$ Nayelhi Saavedra Solano, ${ }^{1}$ Pilar Bernal Pérez, ${ }^{2}$ \\ Lauren Mellor-Crummey, ${ }^{3}$ Marcela Tiburcio Saínz'
}

Artículo original

\section{SUMMARY}

Emotional distress is the subjective sensation of diminishment in wellbeing which manifests itself in a number of unspecific symptoms. It might be a risk factor for the development of mental illness, especially among individuals with psychosocial or biological vulnerability. Recent studies show that primary health care services receive a growing number of patients who suffer distress, but do not fulfill the diagnostic criteria of a mental or physical illness. This phenomenon is more frequent among women. The objective of this paper is to analyze the emotional distress experienced by a group of women who attended primary health care institutions in Mexico City, as well as their perceptions and experiences around the attention received, in order to identify their treatment needs. Data was gathered through techniques and instruments pertaining qualitative methodology. The information was coded and analyzed according to the meaning categorization method developed by Kvale. The results show that the main triggers of emotional distress are associated to daily life worries (lack of money, problems with children, domestic violence, among others). In some cases, it is associated as well with traumatic events, such as violence and sexual abuse in the past or at present. Data also suggest that women do not talk about emotional distress directly during medical consultations and that health care professionals do not identify distress or minimize its importance. These aspects are related to the current characteristics of the service, which lacks a comprehensive approach and a psychosocial point of view.

Key words: Emotional distress, mental health, gender, primary health care.

\section{RESUMEN}

La presencia de malestar emocional -que se define como el conjunto de sensaciones subjetivas que percibe una persona de que su bienestar sufre una merma y que se manifiesta por síntomas inespecíficos- puede constituir un factor de riesgo para la aparición de enfermedades mentales, sobre todo en personas con vulnerabilidades biológicas y psicosociales. Estudios recientes señalan que los servicios de atención primaria reciben un número, cada vez mayor, de personas con malestares que no cubren los criterios diagnósticos de una enfermedad, ya sea mental o física, fenómeno que es más frecuente en las mujeres.

El objetivo de este trabajo es analizar los malestares emocionales de un grupo de mujeres que acude a instituciones de atención primaria de la Ciudad de México, así como sus percepciones y vivencias sobre la atención recibida, con el propósito de identificar necesidades de atención. Para recopilar la información se utilizaron técnicas e instrumentos propios de la metodología cualitativa.

La información se codificó y analizó conforme al método de "categorización de significados" propuesto por Kvale. Los resultados mostraron que los principales detonantes de los malestares emocionales en las participantes se asocian con las preocupaciones que enfrentan cotidianamente (como falta de dinero, problemas con los hijos y violencia intrafamiliar) $y$, en otros casos, con la vivencia de experiencias traumáticas de violencia y abuso sexual, pasadas y presentes. Los datos demuestran también que las mujeres no hablan directamente de su malestar emocional, pero que tampoco lo detecta el personal de salud o que, cuando lo hace, le resta importancia. Lo anterior se relaciona con las condiciones actuales del servicio, que no ofrece una atención integral y adolece de una visión psicosocial.

Palabras clave: Malestar emocional, salud mental, género, primer nivel de atención.

Dirección de Investigaciones Epidemiológicas y Psicosociales del Instituto Nacional de Psiquiatría Ramón de la Fuente Muñiz.

2 Facultad de Medicina, Universidad Nacional Autónoma de México.

Yale University, USA.

Correspondencia: Dra. Shoshana Berenzon Gorn. Investigadora titular de la Dirección de Investigaciones Epidemiológicas y Psicosociales. Instituto Nacional de Psiquiatría Ramón de la Fuente Muñiz. Calz. México-Xochimilco 101, San Lorenzo Huipulco, Tlalpan, 14370, México, D.F. E-mail: berenz@imp.edu.mx 


\section{INTRODUCCIÓN}

El constructo de malestar emocional se ha utilizado ampliamente en la investigación médica y social. ${ }^{1}$ Surge tanto de cuestionamientos realizados desde el campo de la biomedicina -donde se ha observado que muchas manifestaciones emocionales y físicas no reúnen los requisitos para un diagnóstico clínico- ${ }^{2}$ como de posturas más críticas e interpretativas, las cuales enfatizan las sensaciones y representaciones del malestar en el escenario de la vida cotidiana y en la importancia que tienen las variables socioculturales en la construcción del mismo. ${ }^{3-5}$

El malestar emocional es una sensación subjetiva relativa a un bienestar mermado. Se plantea como desazón, incomodidad o "sentirse mal", y aparece como un conjunto de síntomas mal definidos y sin causa orgánica demostrable. ${ }^{6}$ Es una respuesta a distintas situaciones de la vida y del contexto social, como la familia, el trabajo, la comunidad y las actividades de la vida diaria. Algunos de los síntomas y sensaciones asociadas con el malestar emocional son: tristeza, sentimiento de vacío, dolores musculares, de cabeza, o de ambos, insomnio, fatiga, preocupación, nervios e irritabilidad. $^{7}$

Cabe resaltar que la presencia de malestar emocional no es sinónimo de enfermedad mental ni tampoco es una causa directa. Sin embargo, si puede incrementar el riesgo de que se presente en personas con vulnerabilidades biológicas y psicosociales. ${ }^{8}$ Aunado a lo anterior, el malestar emocional genera costos importantes en la salud-bienestar de la población, por lo que trabajar en la delimitación de su campo adquiere especial relevancia para detectar e intervenir de forma adecuada y oportuna. ${ }^{6}$

Estudios recientes coinciden en señalar que en los servicios de atención primaria se recibe un número cada vez mayor de personas que llegan a consulta con molestias similares a las planteadas en la definición de malestar emocional. ${ }^{9-11}$

Así, se estima que entre el 30 y $60 \%$ de todas las visitas en busca de atención primaria se asocian a síntomas que no cubren necesariamente los criterios diagnósticos de una enfermedad, ya sea mental o física, ni pueden confirmarse por medio de pruebas de laboratorio o de gabinete. ${ }^{10,12}$

Este tipo de sensaciones de malestar son mencionadas con mayor frecuencia entre la población femenina. Autores como Velasco, ${ }^{4}$ Muñoz et al. ${ }^{13}$ señalan que, en España, más del $70 \%$ de las personas que presentan este tipo de problemáticas son mujeres. Por ello, proponen el concepto de "síndrome de malestar de las mujeres" como un constructo que incluye el condicionante de género en el abordaje subjetivo y biopsicosocial del malestar emocional.

Vargas et al. ${ }^{14}$ encontraron resultados similares en centros de salud de la Ciudad de México. Su estudio reflejó que el $67 \%$ de las personas entrevistadas presentaron malestar emocional, y que éste fue más frecuente en las mujeres $(70.6 \%)$ que en los hombres (52.5\%).
Las diferencias en la presencia de malestar emocional en uno y otro sexo parecen relacionarse con una forma distinta de enfermar, con una manera diferente de expresar los malestares y con una mayor tendencia de las mujeres a acudir a los centros de salud, lo que hace que sus sensaciones y quejas sean más registrables. ${ }^{15}$

Aunado a lo anterior, diversas investigaciones realizadas en México coinciden en señalar que en la población femenina el malestar emocional se asocia con el mayor número de responsabilidades y obligaciones asignadas a su género, como ser madres, esposas, hijas, cuidadoras y, en muchos casos, también proveedoras. Es decir, en atender las necesidades de los otros antes que las propias. Sobra decir que estas situaciones generan mucha preocupación, tensión y estrés. ${ }^{16-19}$

A pesar de que esta información pone de manifiesto la presencia de malestar emocional en la población femenina que acude a los centros de salud de primer nivel, en la mayoría de los casos este malestar no es detectado o no es tomado en cuenta por el personal que labora en estas instituciones. En todo caso, la atención se orienta a los síntomas y dolores, pero no al origen de los mismos.

Esta manera de abordar el problema responde a ciertos aspectos vinculados con el funcionamiento actual del primer nivel de atención mexicano, como prioridad que se da a las intervenciones enfocadas a los padecimientos físicos, la carencia de programas encaminados al diagnóstico y atención de problemáticas emocionales y la insuficiente capacitación que recibe el personal sobre estos temas. ${ }^{20}$

Asimismo, la corta duración de las consultas y las elevadas cargas burocráticas y administrativas de los servidores impiden profundizar en temas relacionados con la vida cotidiana de cada paciente..$^{20-22}$

Autores como Nimnuan, ${ }^{12}$ Aiarzaguena $^{10}$ y Velasco ${ }^{4}$ plantean que las mujeres con malestar emocional que no reciben una atención adecuada regresarán una y otra vez a consulta, quejándose de los mismos síntomas y/o de otros nuevos porque su demanda no ha sido resuelta. Esta "hiperfrecuentación de los servicios" genera sufrimiento para las pacientes, frustración entre el personal de salud y un importante impacto económico para el sistema sanitario. ${ }^{23}$

Dado lo anterior, el objetivo de este trabajo es explorar el malestar emocional de un grupo de mujeres y sus percepciones y vivencias sobre la atención recibida en instituciones de atención primaria, con el propósito de identificar sus necesidades de atención.

\section{MATERIAL Y MÉTODOS}

El estudio se llevó a cabo en ocho centros de salud de primer nivel ubicados en tres delegaciones de la Ciudad de México. Se realizó una investigación cualitativa que comprendió observaciones y entrevistas semiestructuradas individuales con personal que trabaja en estos centros (35 personas) y con 
usuarios de los mismos (ocho hombres y nueve mujeres). En este trabajo se presenta sólo la información proporcionada por las usuarias de los centros. El trabajo de campo se hizo entre abril de 2012 y septiembre de 2013.

Las entrevistadas se seleccionaron mediante el muestreo teórico o intencionado, ${ }^{24,25}$ en el cual el número de personas no es lo más importante, sino la información que pueda aportar cada una para interpretar el tema de interés. El proceso termina cuando ocurre la saturación, es decir, cuando las entrevistas con personas adicionales no producen ninguna información nueva o relevante para la comprensión del fenómeno. Estamos conscientes de que en este trabajo no se logró una saturación, entre otras causas, por la diversidad de edades de las mujeres entrevistadas. Aun así, la información recolectada constituye un acercamiento a la compresión de este fenómeno.

En este documento se presenta el análisis de la información proporcionada por nueve mujeres que acudieron como pacientes a alguno de los Centros de atención primaria en que se realizó la investigación.

\section{Participantes}

Las edades de las entrevistadas oscilaron entre los 17 y los 70 años. Cuatro de las participantes habían estudiado hasta la primaria; dos, secundaria; dos, preparatoria, y una de ellas estudió una carrera técnica. Todas mencionaron ser de un nivel socioeconómico bajo y vivir en colonias con índices elevados de marginación, violencia y consumo de drogas (cuadro 1).

Los criterios para incorporar a las mujeres al estudio fueron: a) que tuvieran 15 años o más de edad, b) que asistieran al centro de salud de manera regular para poder compartir su experiencia en cuanto a la atención recibida, y c) que aceptaran participar en el estudio.

\section{Procedimientos para la recopilación y el análisis de los datos}

Se realizaron entrevistas semiestructuradas mediante la técnica de entrevista de investigación social, que favorece la producción de un discurso continuo y posee una línea argumental sobre un tema determinado. ${ }^{26}$

Para recopilar la información se elaboró una guía de entrevista que incluyó los siguientes temas clave: 1. Experiencias y utilización de servicios de salud; 2. Salud mental, del cual sólo se incluye la información relacionada con el malestar emocional y las necesidades de atención dentro del centro de salud; 3. Datos sociodemográficos.

El contacto con las usuarias se realizó por dos vías: por medio de las enfermeras y/o trabajadoras sociales de los centros o por invitación directa mientras esperaban su turno para entrar a consulta. Las entrevistas se llevaron a cabo en las salas de espera de los centros de salud en los horarios y días establecidos por las informantes. Se realizaron en una o dos sesiones, dependiendo de la disponibilidad de tiempo de las entrevistadas, y tuvieron una duración aproximada de 90 minutos. Un maestro en salud mental pública y una estudiante de doctorado llevaron a cabo las entrevistas.

\section{Análisis de la información}

Las entrevistas grabadas en audio fueron transcritas textualmente. Las transcripciones se codificaron y analizaron utilizando el método de "categorización de significados" propuesto por Kvale. ${ }^{27}$ Con este método, cada entrevista se codifica en una serie de categorías mutuamente excluyentes, lo que permite estructurar las narraciones en unidades de información que facilitan la comprensión del tema de interés, así como su ocurrencia a lo largo del discurso.

Dos miembros del equipo de investigación, una doctora en antropología y un maestro en salud mental pública, codificaron las entrevistas por separado y las discrepancias en la codificación se resolvieron mediante la discusión y la revisión conjunta de los relatos originales. En un principio, las categorías se definieron a partir de los objetivos del estudio y de los temas abordados en la guía de entrevista. Posteriormente, se hizo una relectura de las entrevistas para extraer nueva información relevante que sugiriera la adición o modificación de las categorías ya identificadas.

Cuadro 1. Características de las participantes

\begin{tabular}{|c|c|c|c|c|c|c|c|}
\hline Seudónimo & Edad & Estado civil & Hijos & Escolaridad & Ocupación & Enf. crónica & Tx psicológico \\
\hline Reyna & 45 & Casada & Sí & Secundaria & Ama de casa & No & No \\
\hline Juana & 49 & Casada & Sí & Primaria & Ama de casa & No & No \\
\hline Susana & 20 & Unión libre & No & Preparatoria incompleta & Ayuda a su mamá en la casa & No & Sí \\
\hline Socorro & 70 & Viuda & Sí & Primaria & Ama de casa & $\begin{array}{l}\text { Diabetes } \\
\text { e hipertensión }\end{array}$ & No \\
\hline Ema & 17 & Soltera & No & Secundaria & Estudiante & No & Sí \\
\hline Berenice & 39 & Separada & Sí & Carrera técnica & En un salón de belleza & No & No \\
\hline Lucila & 65 & Casada & Sí & Primaria & Ama de casa & Hipertensión & Sí \\
\hline Érica & 60 & Casada & Sí & $4^{\circ}$ año de primaria & Ama de casa & Diabetes & No \\
\hline Eréndira & 18 & Soltera & No & Preparatoria & Estudiante & No & No \\
\hline
\end{tabular}


En este trabajo se presentan los resultados de las siguientes tres categorías:

1. Malestar emocional. Aquí se abordan las sensaciones y manifestaciones percibidas por las entrevistadas que las hacen "sentir mal", "incómodas", "intranquilas" o "molestas", y que no encajan dentro de un cuadro clínico específico. Esta categoría se conformó tras analizar las reacciones corporales y sensaciones emocionales asociadas con el problema o la vivencia que detonó el malestar.

2. Percepciones y vivencias de las mujeres en la atención primaria. En esta categoría se analizan las percepciones que tienen las mujeres sobre la atención que reciben o han recibido en los centros de salud.

3. Necesidades de atención. En esta categoría se exploran las necesidades de atención y apoyo, tanto explícito como implícito, identificadas en los relatos de las entrevistadas.

\section{Consideraciones éticas}

El proyecto del que se desprende la información presentada en este documento fue aprobado por el Comité de Ética del Instituto Nacional de Psiquiatría Ramón de la Fuente Muñiz.

Dado que se consideró como un estudio de riesgo mínimo, el consentimiento informado se solicitó sólo de manera verbal. Para ello se proporcionó información detallada sobre los objetivos y las características de la investigación. Asimismo, se informó a las participantes sobre su derecho a retirar su consentimiento en cualquier momento y dejar de participar en el proyecto sin que ello tuviera alguna repercusión en la atención recibida en el centro de salud. También se solicitó autorización para audiograbar las entrevistas. Para preservar la privacidad de las entrevistadas, se utilizaron seudónimos.

\section{RESULTADOS}

Es importante mencionar que las mujeres entrevistadas habían asistido a los centros de salud por diversos motivos, como llevar a sus hijos a consulta, revisión mensual por embarazo, infecciones intestinales y problemas de garganta, tos o gripe. Las mujeres de mediana y avanzada edad también

\footnotetext{
* La conformación de estos grupos obedece a un programa que tiene los objetivos de promover estilos de vida saludables (ejercicio y cambio de dieta); tener un registro continuo del peso, del nivel de glucosa y de la presión sanguínea de los pacientes. Además de realizar acciones específicas como revisión del fondo de ojo y pie diabético, entre otras. Todo lo anterior entre pacientes diagnosticados con algún tipo de diabetes y/o hipertensión. Los pacientes son convocados en el Centro de Salud, un día específico a la semana para realizar ejercicios y recibir pláticas sobre diversos aspectos de estas enfermedades. Los grupos varían en número, la mayoría de las participantes son mujeres de mediana y avanzada edad. La dinámica de cada grupo depende de la cohesión e interés de las asistentes, de tal forma que hay grupos que se reúnen con mayor frecuencia y que realizan otras actividades, como paseos y convivios.
}

asistían al grupo de crónicos-degenerativos.* Aunque ninguna de las participantes habló de los malestares emocionales como motivo de consulta, éstos fueron apareciendo al abordar los distintos temas de la entrevista.

\section{Malestar emocional}

Las participantes manifestaron sentirse intranquilas, nerviosas, irritables, desesperadas y/o con cambios constantes en el humor. Así, fueron frecuentes frases como: "Me siento alterada ya de mis nervios, tengo miedo, siento angustia" y "Me dan ganas de echarme a correr".

Algunas de ellas presentaron quejas físicas, como dolor persistente en todo el cuerpo, cansancio físico, cansancio mental, dolores de cabeza, "bochornos", fallas en la memoria, insomnio, temblor en las manos y presión alta. Asociaron estas sensaciones de malestar con diversas preocupaciones; por ejemplo, los problemas en casa, el exceso de responsabilidades, la falta de recursos económicos y el abuso de sustancias propio o de algún familiar cercano. También señalaron como detonantes de su malestar vivir en el momento violencia intrafamiliar, haber sido víctimas de abuso sexual o habitar en ambientes inseguros.

En el siguiente relato se ejemplifica cómo se entretejen las quejas físicas, las causas o detonantes de la preocupación y la sensación emocional:

Lucila es una mujer de 65 años que acude con frecuencia al centro de salud porque sufre de presión alta, náuseas, dolores en el pecho y "un calor muy feo en la cabeza". Ella relaciona su hipertensión y "sentirse mal y de malas" con el vivir preocupada constantemente. Sus mayores fuentes de preocupación son: que su esposo no trabaje y por tanto se las "vean muy duras para poder sobrevivir"; el alcoholismo de su hijo y la percepción de inseguridad en la zona donde vive porque "se drogan junto a su puerta y hay veces que están ahí toda la noche".

Si bien es cierto que las sensaciones de malestar pueden manifestarse en las mujeres sin importar su edad, también se identificó que algunas de estas sensaciones se asocian con una etapa específica del ciclo vital. Por ejemplo, las adolescentes relacionan su malestar principalmente con problemas escolares, pleitos con los padres, consumo de drogas y/o alcohol y embarazos no deseados.

"Pues le digo que así tenía ganas de salirme de la escuela, corriendo, de golpear a alguien. De hecho ni quería comer, quería llorar, no sé, no quería hacer nada, nada [...] y de estar en mi casa me sentía mal, y de hecho ahí era peor. Yo siento que todo esto era por ver a mis papás pelear y antes peleaban más feo, ahora ya nada más discuten de palabra; pero antes se aventaban cosas, se peleaban, se pegaban." [Ema, 17 años].

La carga de trabajo cotidiano dentro y fuera de casa, los conflictos con los hijos y la pareja y, en algunos casos, hacerse cargo de los padres son las causas más frecuentes de malestar entre las mujeres adultas. Lo anterior se expresa como "sentirse rebasada" o "hasta el tope". 
"Pues es que siempre hay problemas en la casa, de un modo o de otro. Tengo tres hijos y pues cada hijo hace problemas y uno día con día los va viviendo[...] Mi hija de 14 años salió embarazada, ahorita tuvo su bebé y fue un impacto muy fuerte para nosotros. Yo soy la que me encargo de mi papá, tons, a veces me estresa de que[...] como apenas se le enfermó la bebé a mi hija, nos tuvimos que ir al hospital; todo el día anduve por allá y yo me preocupo de mi papá que ni quién le lleve un taco, y si yo no le llevo, pues él se queda de plano sin comer. Entonces son muchas cosas, me siento jbien presionada!" [Reyna, 45 años].

Finalmente, las mujeres de la tercera edad se sienten preocupadas por sus "achaques", ya sean los característicos del envejecimiento o los asociados con enfermedades crónicas como la diabetes. También son frecuentes, en esta etapa de la vida, las sensaciones de soledad y abandono.

"Ahora como he estado enferma, si me da mucho pendiente, digo: 'Ay, yo sola aquí ni quién me vea'. Tengo diabetes, soy hipertensa, estaba yo bien, pero últimamente se me han complicado muchas cosas. Ahora ya tengo colitis, el colesterol alto, los triglicéridos, padezco mucho de la garganta también. Todo eso me pone mal, sentirme enferma, porque, digo, sí me preocupa estar sola y siempre ando con mucho cuidado porque me da miedo caerme, digo: 'Ay, no' me vaya yo romper algo y entonces sí[...]" [Socorro, 70 años].

\section{Percepciones y vivencias en la atención primaria}

Las mujeres entrevistadas tienen mucha claridad sobre el repertorio de servicios y recursos con que cuentan los centros de salud, por lo que la utilización de éstos les resulta muy práctica. Buscan un diagnóstico para dolores orgánicos o enfermedades físicas y esperan recibir un tratamiento, principalmente medicamentos; o bien llevan a sus hijos a vacunarse o quieren afiliarse al Seguro Popular* o al Programa de Gratuidad.**

Las participantes consideran que la consulta médica no es el espacio adecuado para hablar de lo que les preocupa en su día a día, ya que los tiempos de consulta son muy cortos y les parece que los médicos no cuentan con las habilidades y los conocimientos necesarios para otorgarles una adecuada atención.

Además de lo anterior, prefieren no hablar de sus preocupaciones y sufrimientos porque les da vergüenza, "No debo hablar de mi gente, ventilar cosas de la familia", o tienen miedo de que se les regañe y/o juzgue.

Si bien es cierto que ellas no hablan de manera directa sobre sus malestares, también lo es que el personal de salud no siempre les pregunta sobre su vida cotidiana, sus preocupaciones o su estado de ánimo. Perciben que ellos están

* El Seguro Popular (SP) forma parte del Sistema de Protección Social en Salud que entró en vigor en enero de 2004. Ofrece protección financiera a las personas que no son derechohabientes de las instituciones públicas de seguridad social como el IMSS o el ISSSTE. Está dirigido principalmente a los trabajadores no asalariados, a los desempleados y a sus familiares. El SP es coordinando por la Federación y operado por las entidades federativas.

** Gratuidad es la estrategia del Programa de servicios médicos y medicamentos gratuitos, que se inició en julio del 2001. En 2006 fue emitido como ley en el Distrito Federal y en 2007 fue declarado programa social. Está dirigido a las familias no aseguradas, residentes en la Ciudad de México. más interesados en reconocer y tratar los síntomas físicos y concluir rápidamente la consulta antes que conocer sus historias de vida.

Así, pareciera ser que los profesionales de la salud del primer nivel reconocen los malestares emocionales sólo cuando los problemas son muy evidentes y/o es muy clara su asociación con los síntomas físicos.

Susana, de veinte años, que acude al centro de salud porque está embarazada, comenta que siempre que acude a consulta la enfermera la pesa y le toma la presión y que, en una ocasión, ésta vio que sus manos estaban llenas de cicatrices y le pregunto por qué las tenía así. Susana le platicó que se cortaba cuando se desesperaba y cuando sentía que no hacía las cosas bien. La entrevistada menciona que después de escuchar esto la enfermera le dijo que el embarazo en adolescentes causaba depresión y que sería bueno que sacara una cita con la doctora, haciendo referencia a la psicóloga del centro de salud.

Por otro lado, en los casos en que las entrevistadas les confiaron a los médicos sentirse tensas, estresadas, nerviosas o tristes, la respuesta más frecuente que recibieron por parte de ellos fue que les recetaran algún medicamento, desde antiácidos, analgésicos o relajantes hasta fármacos para "dormir" o para "la depresión".

"Él [el doctor] me empezó a controlar la presión con medicamento y me dio las pastillas para dormir porque me dice que tenía yo 'una fuerte depresión', dice 'con esto se va a levantar', y sí, le digo, hasta la fecha las tomo[... ]" [Lucila, 65 años].

Otra recomendación frecuente del personal médico cuando se reconocía la presencia de malestar emocional en las mujeres, especialmente en las de la tercera edad, era que se incorporaran a los grupos de crónicos degenerativos.

"El doctor me recomendó hacer ejercicio, ir al grupo [de enfermedades crónicas], me dijo que me sentiría mejor, y sí, me siento muy bien con el ejercicio; [me ayuda] en el estrés, más que nada en el estrés. También platicamos, las que vamos, echamos relajo, nos estamos riendo; yo me siento bien, platicamos, nos relajamos y salimos bien contentas[...]" [Érica, 60 años].

A partir de los relatos de las entrevistadas se pudo identificar que también existe una atención "informal" que brindan en ocasiones las enfermeras y trabajadoras sociales. Dicha atención se otorga más bien a partir de la experiencia de vida y de la solidaridad de género que desde un conocimiento profesional. Esta dinámica se da gracias a que se han establecido vínculos de amistad y empatía y a que las pacientes se sienten con la confianza de platicar sus problemáticas.

\section{Necesidades de atención}

De manera explícita, las mujeres señalaron la necesidad de contar con un especialista en salud mental, preferentemente un psicólogo en cada centro. Para ellas, los profesionales de la salud mental son los únicos que cuentan con el conocimiento necesario para escuchar y atender los problemas cotidianos que las aquejan. También perciben necesario reci- 
bir información de temas relacionados con el cuidado de los hijos, la depresión, la violencia familiar, las drogas y otros.

Otra necesidad expresada por estas mujeres es que estos servicios especializados estén disponibles en los centros, tanto en el turno matutino como en el vespertino, ya que frecuentemente se les complica acudir en la mañana porque tienen que hacer la comida, llevar a los hijos a la escuela y trabajar, entre otras cosas.

Como se observa, las necesidades explícitas se depositan claramente en los especialistas de la salud mental y descartando la posibilidad de que el personal médico, de enfermería o de trabajo social del primer nivel pueda otorgar este tipo de atención.

Sin embargo, el análisis de los relatos develó necesidades subyacentes que se asocian con la relación cotidiana que se mantiene con el personal del primer nivel y con el funcionamiento del mismo.

En los discursos de las mujeres se percibe la necesidad de que se les preste una escucha más sensible y empática por parte del personal, en especial de los médicos. Esta falta de empatía y sensibilidad a menudo se asocia con características del personal como la edad (al considerar que si son muy jóvenes están menos capacitados) o con el sexo (al sentir mayor confianza con las mujeres).

De lo dicho por las mujeres se desprende que también existe una necesidad de contar con espacios privados, pues sienten cierta incomodidad para hablar de temas "personales e íntimos" en consultorios donde la puerta está abierta por lo regular, donde las paredes son de materiales que no aíslan los sonidos o donde la gente entra y sale constantemente.

\section{DISCUSIÓN}

Los testimonios aquí analizados permiten valorar la perspectiva de las pacientes respecto a cómo se entretejen sus quejas, las causas o detonantes de sus preocupaciones y la sensación de malestar emocional, así como las percepciones $\mathrm{y}$ vivencias en torno a la atención de este último.

Los resultados de otras investigaciones ${ }^{5-7}$ apoyan lo señalado anteriormente, en el sentido de que el malestar emocional en las mujeres sólo se podrá detectar y atender en la medida en que se comprenda que, en su origen, se conjugan diversos factores. Estos factores se asocian con su biografía, con las condicionantes de género y con la forma en que se experimentan y se da significado a las circunstancias socioculturales.

Los datos demuestran también que las mujeres no hablan directamente de su malestar emocional, pero el personal de salud tampoco lo detecta y, cuando lo hace, le resta importancia. Ello se relaciona quizás con las condiciones actuales del servicio otorgado en primer nivel, que no ofrece una atención integral y carece de una visión psicosocial.

Para que se logre brindar una atención adecuada al malestar emocional sería necesario realizar cambios dentro del primer nivel de atención que permitan: a) sensibilizar y capacitar a las autoridades y al personal sobre el impacto de los sucesos de la vida cotidiana en la salud, así como sobre la importancia de escuchar situaciones diferentes a la queja médica o somática, y b) integrar la detección del malestar emocional y su atención como uno de los objetivos del programa de salud mental. ${ }^{28,29}$

Realizar las acciones antes propuestas y escuchar lo que se oculta detrás del síntoma permitiría entender de manera más clara las demandas de las mujeres y dar, por tanto, una atención más acorde con sus necesidades. Con este cambio en la atención se podría disminuir lo que se ha llamado "hiperfrecuentación de los servicios de atención primaria", 4 la que, como ya se mencionó, produce sufrimiento a las pacientes, frustración e impotencia en el personal de salud y un impacto económico para el sistema sanitario, justamente porque la demanda no es resuelta del todo. ${ }^{4,13,23}$

Las características y dinámicas instituidas en los centros de salud en que se realizó este proyecto dificultaron el primer contacto con las usuarias, el establecimiento de fechas y horarios de las entrevistas y el desarrollo óptimo de las mismas. Es necesario buscar mejores estrategias para lograr una mayor participación de las mujeres en el estudio y para que éstas estén dispuestas a dar entrevistas en espacios diferentes al centro de salud.

En trabajos posteriores sería recomendable complementar la información presentada en este artículo con la visión que tiene el personal de los centros de atención primaria respecto del malestar de las mujeres y sus necesidades de atención. Por último, también sería conveniente realizar otras investigaciones enfocadas a un análisis más preciso de la relación entre la presencia de malestar emocional, la falta de reconocimiento y atención del mismo y la "hiprefrecuentación de los servicios de atención primaria" por parte de la población femenina.

\section{AGRADECIMIENTOS}

Las autoras y el autor agradecen y expresan su reconocimiento a la Secretaría de Salud del Distrito Federal y en especial a las personas entrevistadas que compartieron sus experiencias para hacer posible este trabajo. El proyecto de investigación base de estos resultados recibió financiamiento del Consejo Nacional de Ciencia y Tecnología (SEP-2011-C01-166588) y del Programa de Igualdad entre Hombres y Mujeres 2013.

\section{REFERENCIAS}

1. Salazar J, Sempere E. Malestar emocional: Manual práctico para una respuesta en atención primaria. España: Generalitat Valencia; 2012.

2. Rivas M, Nuevo R, Ayuso-Mateos J. Depresión subclínica en España: prevalencia e impacto sobre la salud. Revista Psiquiatría Salud Mental 2011;4(3):144-149.

3. Castro R. La vida en la adversidad: el significado de la salud y la reproducción de la pobreza. Primera edición. Cuernavaca, Mor.: UNAM/ Centro Regional de Investigaciones Multidisciplinarias; 2000. 
4. Velasco AS. Atención biopsicosocial al malestar de las mujeres. Intervención en atención primaria de salud. Madrid, Esp.: Instituto de la Mujer; 2006.

5. Burin M, Moncarz E, Velázquez S. El malestar de las mujeres. La tranquilidad recetada. Buenos Aires: Paidós; 1990.

6. Cucco M. ProCC: Una propuesta de intervención sobre los malestares de la vida cotidiana. Del destino social a la precariedad narcisista. Primera edición. Buenos Aires, Arg.: Atuel; 2006.

7. Tubert S. Construcción cultural de la feminidad. Salud mental y género. Aspectos psicosociales diferenciales en la salud de las mujeres. Madrid: Instituto de la Mujer. Ministerio de Trabajo y Asuntos Sociales; 2000.

8. Medina-Mora, Berenzon S. Salud mental y adicciones. Libro $\mathrm{N}^{\circ} 5$ de la serie Agenda Ciudadana de Ciencia, Tecnología e Innovación. Primera edición. México: Academia Mexicana de Ciencias; 2013.

9. Vázquez JL; Wilkinson G, Williams P, Díez J et al. Mental health and medical consultation in primary care settings. Psychological Medicine 1990;20:681-694.

10. Airzaguena J, Grandes G, Alonso-Airbiol I, del Campo J et al. Abordaje biopsicosocial de los pacientes somatizadores en las consultas de atención primaria: un estudio piloto. Aten Prim 2002;29(9):558-561.

11. Vargas B, Villamil V, Pérez J. Los modelos de atención primaria en salud mental: el caso de México. Atención Primaria 2012;44(7):441-442.

12. Nimnuan C, Rabe-Hesketh S, Wesseley S, Hotopf M. "How many functional somatic syndromes?" J Psychosom Res 2001;51(4):549-557.

13. Muñoz MA, De Casas M, Cobo R, Fontcuberta J et al. Malestar psicosocial de la mujer: Experiencia en una intervención grupal en atención primaria. Archivos Medicina Familiar 2008;10(3):96-100.

14. Vargas B, Villamil V, Rodríguez C, Pérez J et al. Validación de la escala Kessler 10 (K-10) en la detección de depresión y ansiedad en el primer nivel de atención. Propiedades psicométricas. Salud Mental 2011;34(4)323-331.

15. Fugita F, Diener E, Sandick E. Gender differences in negative affect and well-being: The case for emotional intensitive. J Personality Social Pshycology 1991;61:427-434.
16. Salgado de Snyder N, Diaz M, Ojeda V. The prevalence of nervios and associated symptomatology among inhabitants of Mexican rural communities. Cultural Medicine Psychiatry 2000;24:453-470.

17. Medina-Mora ME, Rojas E. Mujer, pobreza y adicciones. Perinatol Reprod Hum 2003;17:230-244.

18. Lara MA, Acevedo M, Berenzon S. La depresión femenina vista desde la subjetividad de las mujeres. Cad Saúde Pública 2004;20(3):818-828.

19. Rocha T, Cruz C. Barreras estructurales y subjetivas en la transición de roles de mujeres mexicanas y su malestar emocional. Acta Colombiana Psicología 2013;16(1):123-135.

20. Ruíz-Gómez A, Mercado-Martínez F, Pere-Aceves M. La atención a la enfermedad crónica en los servicios públicos de salud. La perspectiva de los profesionales y los legos. Investigación Salud 2006;1(VIII):23-30.

21. Herrera C, Agoff C. Dilemas del personal médico ante la violencia de pareja en México. Cad Saúde Pública 2006;11(22):2349-2357.

22. Juárez C. Aunque la casa se queme que no salga el humo. Experiencias y atención a la violencia de pareja en México. Primera edición. Quito, Ecuador: Abya-Yala; 2012.

23. Rodríguez MR. Abordaje del paciente hiperfrecuentador de servicios en atención primaria: un acercamiento desde la teoría. Rev Gerenc Polit Salud 2012;11(22):43-55.

24. Taylor S, Bodgan R. Introducción a los Métodos Cualitativos de Investigación. Tercera edición. Buenos Aires: Paidós; 1984.

25. Ruíz-Olabuenaga J. Metodología de la investigación cualitativa. Primera edición. España: Universidad de Deusto; 2012.

26. Alonso L. Sujeto y discurso: El lugar de la entrevista abierta en las prácticas de la sociología cualitativa. En: Delgado JM, Gutiérrez J (eds). Métodos y técnicas de investigación en ciencias sociales. Madrid: Síntesis; 1995.

27. Kvale S. InterViews. An introduction to qualitative research interviewing. Primera edición. California: SAGE Publications; 1996.

28. O'Malley A, Forrest C, O'Malley P. Low-income women's priorities for primary care: a qualitative study. J Fam Pract 2000;49(2):141-146.

29. Lamb J1, Bower P, Rogers A, Dowrick C et al. Access to mental health in primary care: a qualitative meta-synthesis of evidence from the experience of people from 'hard to reach' groups. Health (Londres) 2012;16(1):76-104.

Artículo sin conflicto de intereses 